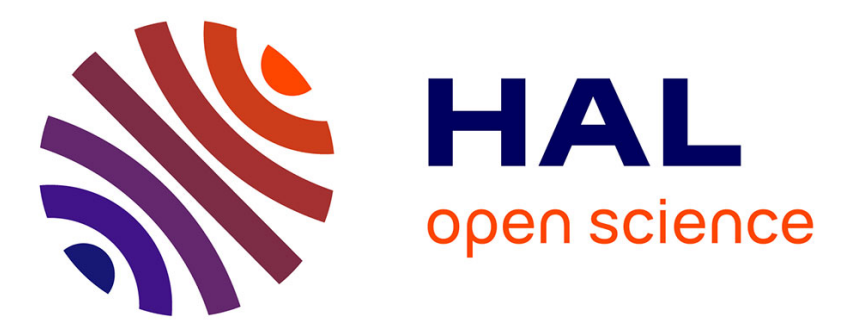

\title{
Propriétés magnétiques des ions d3 dans des structures lamellaires
}

Gilles Le Flem, Claude Delmas, François Ménil, Michel Niel, Christian Cros, Claude Fouassier, M. Pouchard

\section{- To cite this version:}

Gilles Le Flem, Claude Delmas, François Ménil, Michel Niel, Christian Cros, et al.. Propriétés magnétiques des ions d3 dans des structures lamellaires. Journal de Physique Colloques, 1977, 38 (C7), pp.262-265. 10.1051/jphyscol:1977750 . jpa-00217253

\section{HAL Id: jpa-00217253 https://hal.science/jpa-00217253}

Submitted on 1 Jan 1977

HAL is a multi-disciplinary open access archive for the deposit and dissemination of scientific research documents, whether they are published or not. The documents may come from teaching and research institutions in France or abroad, or from public or private research centers.
L'archive ouverte pluridisciplinaire $\mathbf{H A L}$, est destinée au dépôt et à la diffusion de documents scientifiques de niveau recherche, publiés ou non, émanant des établissements d'enseignement et de recherche français ou étrangers, des laboratoires publics ou privés. 


\title{
PROPRIÉTÉS MAGNETIQUES DES IONS $\mathbf{d}^{3}$ DANS DES STRUCTURES LAMELLAIRES
}

\author{
G. LE FLEM, C. DELMAS, F. MENIL, M. NIEL, C. CROS, C. FOUASSIER et M. POUCHARD \\ Laboratoire de Chimie du Solide du C.N.R.S., Université de Bordeaux I, \\ 351 cours de la Libération, 33405 Talence, Cedex, France
}

\begin{abstract}
Résumé. - Les propriétés magnétiques des phases bidimensionnelles $\mathrm{VX}_{2}(\mathrm{X}=\mathrm{Cl}, \mathrm{Br}, \mathrm{I})$ et $\mathrm{ACrO}_{2}(\mathrm{~A}=\mathrm{Li}, \mathrm{Na}, \mathrm{K})$ ont été étudiées entre 4,2 et $800 \mathrm{~K}$. Les intégrales d'échange ont été calculées sur la base d'un modèle de Heisenberg bidimensionnel. Les résultats obtenus impliquent de fortes interactions antiferromagnétiques à l'intérieur des couches. La nature des couplages est discutée.

Abstract. - The magnetic properties of $2 \mathrm{D}$ antiferromagnetic $\mathrm{VX}_{2}(\mathrm{X}=\mathrm{Cl}, \mathrm{Br}, \mathrm{I})$ and $\mathrm{ACrO}_{2}$ $(\mathrm{A}=\mathrm{Li}, \mathrm{Na}, \mathrm{K}$ ) phases have been studied in the temperature range $4.2-800 \mathrm{~K}$. The exchange integrals have been calculated according to a Heisenberg two-dimensional model. The results obtained involve strong antiferromagnetic interactions inside the layers. The nature of the couplings is discussed.
\end{abstract}

Le caractère anisotrope de la structure cristalline d'un matériau n'est pas sans influence sur ses propriétés magnétiques. C'est ainsi que la compréhension des phénomènes critiques et l'établissement des diagrammes de phase magnétique ont permis de dégager depuis quelques années le concept de dimensionalité des interactions magnétiques.

Il s'agit de déterminer les conditions d'existence d'une température d'ordre et de calculer la valeur des intégrales d'échange en prenant en considération la dimensionalité de la structure (1D : chaînes, 2D : couches, $3 \mathrm{D}$ : tridimensionnelle), la topologie des ions magnétiques et le nombre de composantes $n$ du paramètre d'ordre ( $n=1$ : Ising, $n=2 \mathrm{XY}, n=3$ : Heisenberg).

Cette nouvelle approche des relations entre structure et propriétés magnétiques peut être illustrée par l'étude des deux familles de composés lamellaires $\mathrm{VX}_{2}(\mathrm{X}=\mathrm{Cl}, \mathrm{Br}, \mathrm{I})$ et $\mathrm{ACrO}_{2}(\mathrm{~A}=\mathrm{Li}, \mathrm{Na}, \mathrm{K})$ dont les ions magnétiques $\mathrm{V}^{2+}$ et $\mathrm{Cr}^{3+}$ possèdent le même nombre quantique de $\operatorname{spin} S=3 / 2$ et la même topologie au sein de leur structure cristalline respective.

Les dihalogénures $\mathrm{VCl}_{2}, \mathrm{VBr}_{2}$ et $\mathrm{VI}_{2}$ sont caractérisés par une structure à couches de type $\mathrm{CdI}_{2}$. Le réseau peut être décrit comme constitué par des feuillets [VX $\mathrm{VX}_{2}$ ] d'octaèdres mettant en commun six de leurs arêtes et se développant perpendiculairement à l'axe c (figure 1). Ces feuillets se déduisent les uns des autres par simple translation le long de l'axe $c$. Les ions $\mathrm{V}^{2+}$ situés au sein des plans successifs sont distants d'une valeur égale au paramètre $a$. Entre plans 001 adjacents, les distances séparant les ions
$\mathrm{V}^{2+}$ les plus proches sont égales au paramètre $c$ (tableau 1) [1].

TABleau I

Paramètres des phases $V X_{2}[1]$

\begin{tabular}{|c|c|c|c|}
\hline & $\mathrm{VCl}_{2}$ & $\mathrm{VBr}_{2}$ & $\mathrm{VI}_{2}$ \\
\hline $\mathrm{a}(\stackrel{\circ}{\mathrm{A}})$ & 3,601 & 3,776 & 4,057 \\
\hline $\mathrm{c}(\stackrel{\circ}{\mathrm{A}})$ & 5,835 & 6,229 & 6,750 \\
\hline
\end{tabular}

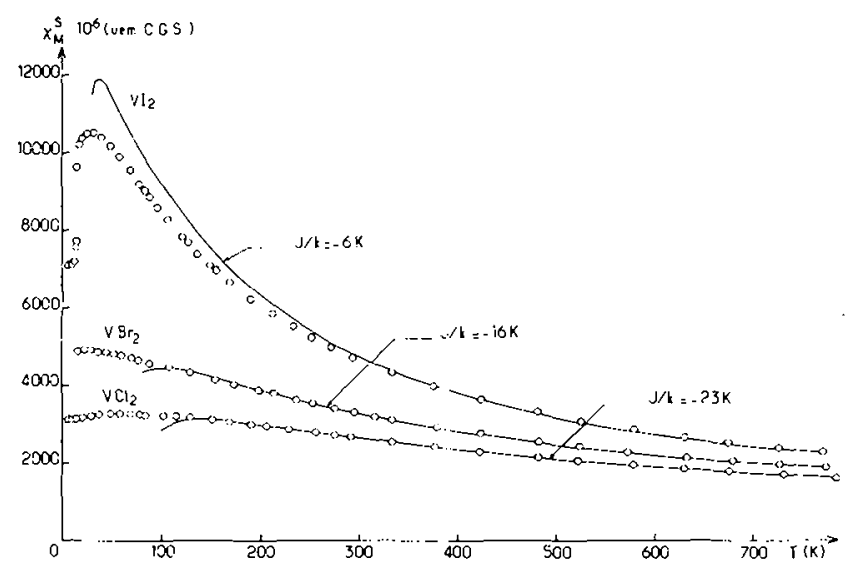

FIG. 1. - Structure des phases $\mathbf{V X}_{2}$.

Les oxydes $\mathrm{ACrO}_{2}$ appartiennent au type structural $\mathrm{NaFeO}_{2} \alpha$ caractérisé par un empilement $\mathrm{ABCABC}$ des atomes d'oxygène. La structure est donc constituée 
de feuillets $\left(\mathrm{CrO}_{2}\right)_{n}^{-}$d'octaèdres à arêtes communes séparés par des couches de cations alcalins (figure 2). Les ions $\mathrm{Cr}^{3+}$ au sein des couches oxygénées sont distants d'une valeur égale au paramètre $a$. Les distances $\mathrm{Cr}$-Cr intercouche entre premiers voisins sont

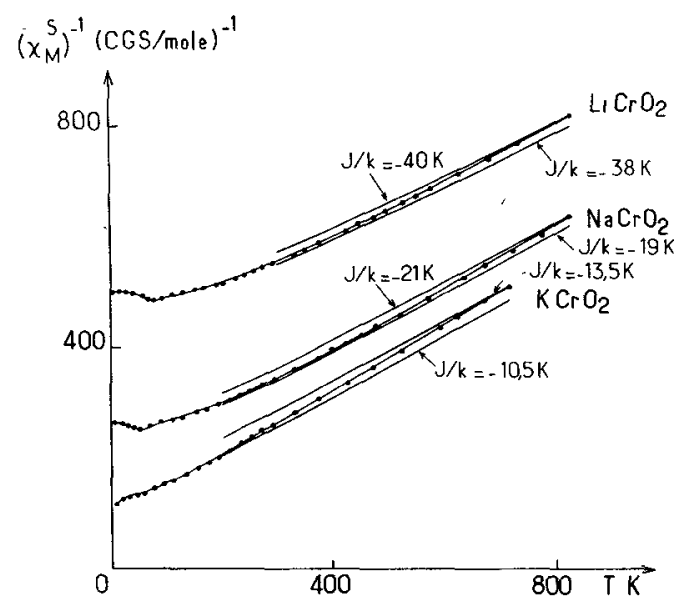

FIG. 2. - Structure des phases $\mathrm{ACrO}_{2}$.

TABLEAU II

Evolution des paramètres cristallographiques des phases $\mathrm{ACrO}_{2}[2]$

\begin{tabular}{|c|c|c|c|}
\hline & $\mathrm{LiCrO}_{2}$ & $\mathrm{NaCrO}_{2}$ & $\mathrm{KCrO}_{2}$ \\
\hline $\mathrm{a}(\AA)$ & 2,90 & 2,97 & 3,02 \\
\hline $\mathrm{c}(\AA)$ & 14,43 & 15,94 & 17,76 \\
\hline $\begin{array}{c}\mathrm{d}_{\mathrm{Cr}-\mathrm{Cr}}(\stackrel{\circ}{\AA}) \\
\text { 2ntracouche }\end{array}$ & 2,90 & 2,97 & 3,02 \\
\hline $\begin{array}{c}\mathrm{d}_{\mathrm{Cr}-\mathrm{Cr}}(\stackrel{\circ}{\mathrm{A}}) \\
\text { intercouche }\end{array}$ & 5,02 & 5,58 & 6,17 \\
\hline
\end{tabular}

nettement supérieures (tableau II) [2]. Les susceptibilités magnétiques de tous ces composés ont été mesurées de 4,2 à $800 \mathrm{~K}$. Pour tenir compte de l'effet Zeeman du second ordre et du couplage spin orbite, les valeurs mesurées de la susceptibilité magnétique $\chi_{M}^{\text {mes }}$ ont été corrigées à l'aide de la relation suivante établie pour un terme fondamental ${ }^{4} \mathrm{~A}_{2 \mathrm{~g}}$ :

$$
\chi_{\mathrm{M}}^{\mathrm{s}}=\frac{\chi_{\mathrm{M}}^{\mathrm{mes}}-8 k^{2} N \mu_{\mathrm{B}}^{2} / \Delta}{1-8 k^{2} \lambda_{0} / \Delta} .
$$

Dans cette expression $N$ est le nombre d'Avogadro, $\mu_{\mathrm{B}}$ le magnéton de Bohr, $\lambda_{0}$ le paramètre de couplage spin orbite, $k$ le facteur de liaison déterminé par RPE, $\Delta$ l'éclatement dû au champ de ligande, déterminé par des mesures optiques (tableau III) [3].

Les courbes $\chi_{\mathrm{M}}^{\mathrm{S}}=f(T)$ pour les phases $\mathrm{VX}_{2}$ et $\left(\chi_{\mathrm{M}}^{\mathrm{S}}\right)^{-1}=f(T)$ pour les phases $\mathrm{ACrO}_{2}$ sont représentées aux figures 3 et 4 . Les valeurs de la constante de Curie, du moment effectif, de la température de Curie paramagnétique correspondantes sont ras-
TABleAU III

Paramètres de corrections des susceptibilités magnétiques [1,2]

\begin{tabular}{|c|c|c|c|c|c|c|}
\hline & $\mathrm{VCl}_{2}$ & $\mathrm{VBr}_{2}$ & $\mathrm{VI}_{2}$ & $\mathrm{~L}_{1} \mathrm{CrO}_{2}$ & $\mathrm{NaCrO}_{2}$ & $\mathrm{KCrO}_{2}$ \\
\hline $\mathrm{g}^{\prime}$ & 1,97 & 1,99 & 2,02 & 1,98 & 1,98 & 1,98 \\
\hline $\mathrm{k}$ & 0,78 & 0,43 & 0 & 0,7 & 0,7 & 0,7 \\
\hline$\Delta\left(\mathrm{cm}^{-1}\right)$ & 9.200 & 8.600 & 7.900 & 17.500 & 17,100 & 16.600 \\
\hline
\end{tabular}

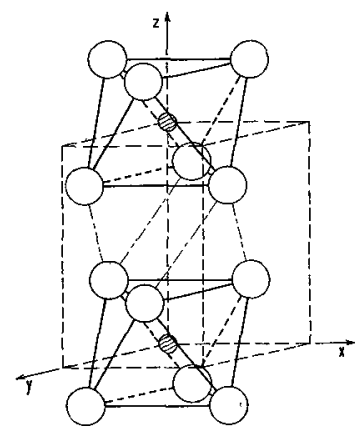

(a)

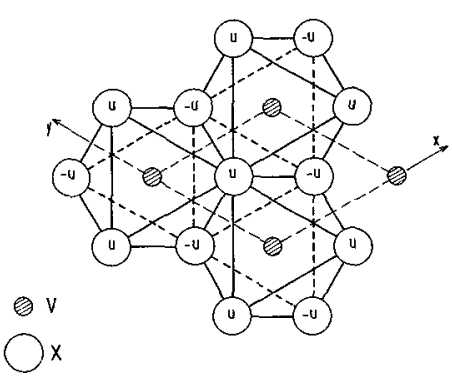

(b)
FIG. 3. - Variation thermique des susceptibilités observées et calculées pour les phases $\mathrm{VX}_{2}$.

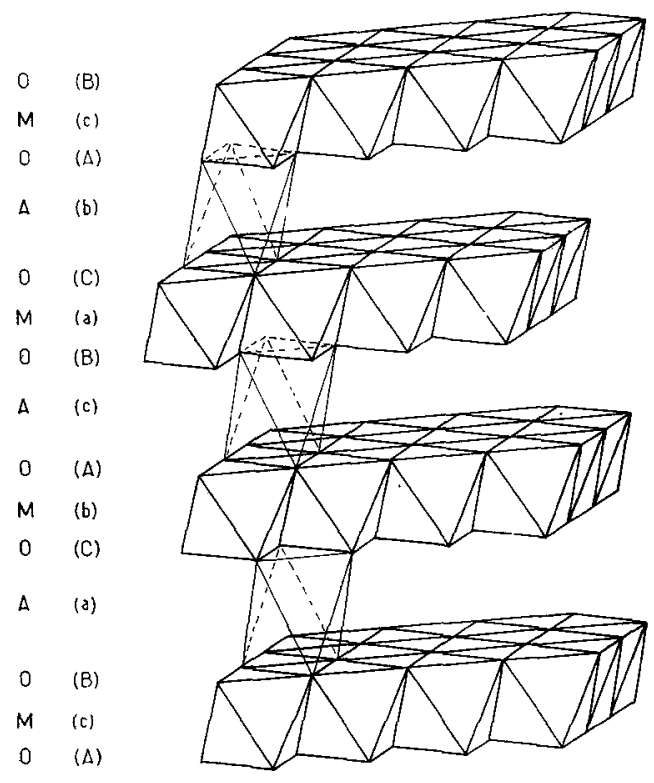

FIG. 4. - Variation thermique des susceptibilités réciproques observées et calculées pour les phases $\mathrm{ACrO}_{2}$.

semblées au tableau IV. Les valeurs de $\mu_{\text {eff }}$ sont comparées à celles calculées dans l'hypothèse de la seule contribution de spin pour $S=3 / 2$.

Calcul de l'intégrale d'échange. - La morphologie des deux types structuraux impliqués laissait prévoir que les couplages magnétiques à l'intérieur des plans 
TABLEAU IV

Données magnétiques expérimentales et théoriques relatives aux phases $\mathrm{VX}_{2}$ et $\mathrm{ACrO}_{2}$.

\begin{tabular}{|c|c|c|c|c|c|c|}
\hline & $\mathrm{VCl}_{2}$ & $\mathrm{VBr}_{2}$ & $\mathrm{VI}_{2}$ & $\mathrm{LiCrO}_{2}$ & $\mathrm{NaCrO}_{2}$ & $\mathrm{KCrO}_{2}$ \\
\hline$C_{M}$ & 1,96 & 2,07 & 2,07 & 1,83 & 1,75 & 1,72 \\
\hline$\mu_{\mathrm{eff}}\left(\mu_{B}\right)$ & 3,96 & 4,07 & 4,07 & 3,82 & 3,74 & 3,71 \\
\hline${ }_{9} \sqrt{\mathrm{S}(\mathrm{S}+1)}\left(\mu_{B}\right)$ & 3,815 & 3,854 & 3,912 & 3,83 & 3,83 & 3,83 \\
\hline${ }_{\theta_{p}(K)}$ & -437 & -335 & -143 & -680 & -280 & -155 \\
\hline
\end{tabular}

étaient nettement supérieurs aux couplages entre plans. D'autre part les valeurs des températures de Curie paramagnétiques supposaient des interactions antiferromagnétiques (AF) prépondérantes.

Ces deux hypothèses nous ont conduits à déterminer les intégrales d'échange intraplanaires des phases $\mathrm{VX}_{2}$ et $\mathrm{ACrO}_{2}$ à partir des calculs de Rushbrooke et Wood pour des interactions AF bidimensionnelles dans le cas de mailles triangulaires [4].

Pour ce modèle la susceptibilité $\chi_{M}^{S}$ est liée à la température $T$ par la relation :

où

$$
\frac{N g^{2} \mu_{\mathbf{B}}^{2}}{\chi_{\mathrm{M}}^{\mathbf{S}} J}=3\left[x+4+\frac{3,20}{x}-\frac{2,816}{x^{2}}+\frac{0,08}{x^{3}}+\frac{3,45}{x^{4}}-\frac{3,99}{x^{5}}\right]
$$

$$
x=\frac{k T}{J S(S+1)} .
$$

Le tableau $\mathrm{V}$ présente les résultats obtenus qui sont illustrés également par les figures 3 et 4 .

\section{TABLEAU V}

Intégrales d'échange intraplanaires des phases $\mathrm{VX}_{2}$ et $\mathrm{ACrO}_{2}$

\begin{tabular}{|c|c|c|c|c|c|c|}
\hline & $\mathrm{VCl}_{2}$ & $\mathrm{VBr}_{2}$ & $\mathrm{VI}_{2}$ & $\mathrm{LiCrO}_{2}$ & $\mathrm{NaCrO}_{2}$ & $\mathrm{KCrO}_{2}$ \\
\hline $\mathrm{J} / \mathrm{k}(\mathrm{K})$ & -26 & -16 & -6 & -40 & -20 & -12 \\
\hline
\end{tabular}

Interprétations des résultats obtenus. -- A basse température la variation thermique de la susceptibilité magnétique de $\mathrm{VCl}_{2}, \mathrm{VBr}_{2}$ et $\mathrm{KCrO}_{2}$ est très faible. En revanche pour $\mathrm{VI}_{2}, \mathrm{LiCrO}_{2}$ et $\mathrm{NaCrO}_{2}$ elle présente un maximum respectivement à 25,75 et $50 \mathrm{~K}$, ce qui suppose l'existence d'une température critique $T_{\mathrm{C}}$ au-dessous de laquelle s'établirait l'ordre à grande distance.

D'une manière générale dans un modèle de Heisenberg l'existence d'une différence entre $T_{\mathrm{C}}$ et le maximum de susceptibilité reflète la présence d'un ordre à courte distance au-dessus de $T_{\mathrm{C}}$. Cet écart s'accroît lorsque la dimensionalité du système s'abaisse [5]. Pour $\mathrm{VI}_{2}, \mathrm{LiCrO}_{2}$ et $\mathrm{NaCrO}_{2}$ les températures $T_{\mathrm{C}}$ sont proches respectivement de $15 \mathrm{~K}, 60 \mathrm{~K}$ et $40 \mathrm{~K}$.

Le modèle de Heisenberg idéal exclut l'existence d'une température critique différente de zéro pour un système bidimensionnel. Un écart à l'idéalité du modèle peut résulter soit de l'existence d'une anisotropie caractérisée par un champ $H_{\mathrm{A}}$ soit d'un couplage $J^{\prime}$ non négligeable entre plans.

L'influence de l'anisotropie a été discutée par De Jongh, Bloembergen et Colpa [6]. Ces auteurs montrent que tout modèle idéal où intervient un champ $H_{\mathrm{A}}$ se traduit par l'existence d'une température
$T_{\mathrm{C}}$ bidimensionnelle toujours supérieure à la température $T_{\mathrm{C}}^{(2)}$ définie d'après Stanley et Kaplan par la relation

$$
T_{\mathrm{C}}^{(2)}=\frac{1}{5}(Z-1)[2 S(S+1)-1] \frac{|J|}{k}
$$

où $Z$ représente le nombre de premiers voisins dans le plan [7].

Les valeurs de $T_{\mathrm{C}}^{(2)}$ calculées pour toutes les phases étudiées excluent l'hypothèse d'une anisotropie notable, puisqu'elles sont nettement supérieures aux températures où les susceptibilités passent par des maxima (tableau VI).

\section{TABLEAU VI}

Températures de Stanley-Kaplan des phases $\mathrm{VX}_{2}$ et $\mathrm{ACrO}_{2}$

\begin{tabular}{|l|c|c|c|c|c|c|}
\hline & $\mathrm{VCI}_{2}$ & $\mathrm{VBr}_{2}$ & $\mathrm{VI}_{2}$ & $\mathrm{~L}_{1} \mathrm{CrO}_{2}$ & $\mathrm{NaCrO}_{2}$ & $\mathrm{KCrO}_{2}$ \\
\hline $\mathrm{T}_{\mathrm{C}}{ }^{(2)}(\mathrm{K})$ & 143 & 98 & 42 & 254 & 130 & 78 \\
\hline
\end{tabular}

En revanche l'influence d'une interaction interplanaire $J^{\prime}$ se traduira par un certain ordre tridimensionnel qui peut être confirmé par la mesure du coefficient critique $\beta$ caractérisant l'évolution de l'aimantation du sous-réseau au voisinage de la température d'ordre. A titre d'exemple, nous avons déterminé ce coefficient pour la phase $\mathrm{LiCr}_{0,99} \mathrm{Fe}_{0,01} \mathrm{O}_{2}$ à partir d'une étude par résonance Mössbauer. La valeur obtenue $\beta=0,25$ est à comparer aux valeurs théoriques calculées pour des modèles bidimensionnels $(\beta \simeq 0,125)$ ou tridimensionnels $(\beta \simeq 0,33)$ [8]. Elle suggère la présence simultanée de corrélations magnétiques bi- et tridimensionnelles en dessous du point d'ordre. 
Les valeurs des intégrales d'échange peuvent être interprétées à l'aide de la théorie du superéchange. Dans toutes les phases considérées les atomes de vanadium ou de chrome sont au centre d'octaèdres liés à leurs voisins par six arêtes et l'angle métalligande-métal diffère peu de $90^{\circ}$. Trois types d'interactions intraplanaires sont à considérer :

- un couplage AF par recouvrement direct des orbitales $t_{2 \mathrm{~g}}$,

- des couplages $F$ par superéchange de type délocalisation $t_{2 \mathrm{~g}}-p-e_{\mathrm{g}}$,

- des couplages par superéchange de type corrélation $t_{2 \mathrm{~g}}-p-e_{\mathrm{g}}: \mathrm{F} ; e_{\mathrm{g}}-s-e_{\mathrm{g}}: \mathrm{AF} ; e_{\mathrm{g}}-p \sigma-p \sigma^{\prime}-e_{\mathrm{g}}: \mathrm{F}$ et $t_{2 \mathrm{~g}}-p \pi-p \pi^{\prime}-t_{2 \mathrm{~g}}: \mathrm{F}$.

Pour la séquence $\mathrm{VCl}_{2}, \mathrm{VBr}_{2}, \mathrm{VI}_{2}$, le renforcement des interactions ferromagnétiques coïncide avec une augmentation des distances $\mathrm{V}-\mathrm{V}$ et un accroissement du caractère covalent de la liaison métal-halogène. L'évolution de $J / k$ s'explique par l'affaiblissement des couplages directs AF très sensibles aux distances $\mathrm{V}-\mathrm{V}$ et par l'extension des orbitales $p$ plus importante que celle des orbitales $s$ lors du passage du chlore à l'iode. Le couplage $t_{2 \mathrm{~g}}-p-e_{\mathrm{g}}$ ainsi que les couplages par doubles corrélations qui sont ferromagnétiques, prendraient alors une importance accrue par rapport aux interactions $\mathrm{AF} e_{\mathrm{g}}-s-e_{\mathrm{g}}$.

Pour les phases $\mathrm{ACrO}_{2}$ les interactions directes $\mathrm{AF}$ sont prédominantes et l'évolution de $J / k$ est liée à l'accroissement de la distance $\mathrm{Cr}-\mathrm{Cr}$ avec l'élément alcalin. Les interactions par superéchange ne varient pas sensiblement : à l'augmentation de la covalence de la liaison $\mathrm{Cr}-\mathrm{O}$ avec le caractère électropositif de l'alcalin s'oppose une dilatation du réseau résultant de l'accroissement de la taille de l'alcalin.

On peut aussi comparer l'évolution des interactions interplanaires pour ces deux familles de composés : l'augmentation de la distance $\mathrm{Cr}-\mathrm{Cr}$ intercouche coïncide avec la diminution de la température d'ordre observée dans les phases $\mathrm{ACrO}_{2}$ lorsqu'on passe du lithium au potassium. En revanche dans le cas des halogénures de vanadium, l'établissement d'un ordre à grande distance n'est observable uniquement que pour $\mathrm{VI}_{2}$ tout au moins au-dessus de $4,2 \mathrm{~K}$. Ce phénomène est à attribuer au caractère très covalent des liaisons vanadium-iode dont la conséquence est l'existence de fortes interactions interplanaires V-I-I-V par super super-échange.

\section{Bibliographie}

[1] NIEL, M., Thèse de doctorat ès sciences, Université de Bordeaux I, 1976

[2] Delmas, C., Thèse de doctorat ès sciences, Université de Bordeaux I, 1976.

[3] Figals, B. N., Introduction to Ligand Fields (Intersciences, Publ. New York) 1961.

[4] Rushirooke, G. S. et Wood, J. P., Mol. Phys. 6 (1963) 409.
[5] de Jongh, L. J. et Miedema, A. R., Adv. Phys. 23 (1974) 1. [6] de Jongh, L. J., Bloembergen, P. et Colpa, J. H. P., Physica 58 (1973) 305

[7] Stanley, H. E. et Kaplan, T. A., Phys. Rev. Lett. 17 (1966) 913.

[8] FrsCher, M. E., Rep. Prog. Phys. 30 (1967) 615. 